\title{
Magnetoterapia: é possível este recurso fazer parte da rotina do fisioterapeuta brasileiro?
}

Magnetotherapy: can this resource be part of Brazilian physiotherapist routine?

Patrícia Froes Meyer', Amanda Paiva e Silva Cavalcanti', Eliane Maria da Silva', Rodrigo Marcel Valentim da Silva', Leandro de Souza Costa', Oscar Ariel Ronzio ${ }^{2}$

\section{Resumo}

0 uso de campos eletromagnéticos pulsados (CEMP) é uma das terapias frequentemente utilizadas pelo fisioterapeuta para tratamento de seus pacientes com diferentes patologias e em diversos países, exceto no Brasil. Devido ao longo tempo de utilização em todos os continentes e a quantidade de publicações existentes sobre o tema, busca-se compreender o motivo de essa técnica não estar presente na rotina de tratamento das clínicas de fisioterapia brasileiras. Para tentar responder a essa questão, realizou-se uma revisão sistemática na base de dados do Pubmed, de 1968 a 2010 (42 anos), com as palavras-chave "PEMF" e "Pulsed Electromagnetic Fields", encontrando-se, respectivamente, 274 e 899 resultados. Após esse levantamento, foram selecionados 36 artigos do período de 1981 a 2008 hand made, além de consulta a 6 livros. Após a coleta de dados, perceberamse resultados positivos no tecido ósseo, em relação à consolidação de fraturas e a osteoporose, devido à sua ação piezoelétrica. 0s efeitos antiinflamatórios e regenerativos também são citados. Portanto, o conhecimento desse recurso no Brasil deve ser despertado em todos os profissionais de saúde que compõem as equipes multidisciplinares de assistência, para que o tratamento possa fazer parte da rotina de trabalho dos fisioterapeutas, beneficiando pacientes de todas as faixas etárias e portadores de diferentes problemas. Sugere-se a fabricação de aparelhos geradores de PEMF por empresas nacionais para diminuir custos e facilitar o acesso a esse agente físico.

Palavras-chave: Terapia de campo magnético; modalidades de fisioterapia; terapêutica.

\begin{abstract}
The use of pulsed electromagnetic fields (PEMF) is one of the therapies frequently used by physiotherapists for treating different patients' pathologies in several countries, with exception of Brazil. Due to the long time of its use in all continents and the numerous publications on this subject, the reason why this technique is not present in the routine of Brazilian physiotherapy clinics should be analyzed. In order to answer this question, a systematic review on the Pubmed data was performed, considering the period from 1968 to 2010 (42 years), with the keywords "PEMF" and "Pulsed Electromagnetic Fields", yielding, respectively, 274 and 899 results. After this survey, additional 36 articles within the period from 1981 to 2008 were selected on a hand made approach, besides the research in 6 books. Following data collection, positive results were verified on bone tissue, regarding fracture consolidation, and osteoporosis, due to piezoelectric action. Anti-inflammatory and regenerative effects were also cited. Therefore, the knowledge of this resource in Brazil should be awakened in all health professionals from multidisciplinary assistance teams, so the treatment can be part of physiotherapists' routine of work, benefiting patients of all ages and with different problems. The manufacturing of a PEMF device by national companies is suggested in order to reduce costs and to facilitate the access to this physical agent.
\end{abstract}

Keywords: Magnetic field therapy; physical therapy modalities; therapeutics.

Recebido: $18 / 2 / 2010$

Revisado: $9 / 8 / 2010$

Aprovado: $17 / 1 / 2011$

\footnotetext{
Trabalho realizado no Departamento de Fisioterapia da Universidade Potiguar (UnP), Natal (RN), Brasil

Universidade Potiguar (UnP), Natal (RN), Brasil

2 Universidad Maimónides, Universidad Favaloro, Universidad del Salvador, Universidad de Morón, Argentina

Endereço para correspondência: Patrícia Froes Meyer - Rua Maxarranguape 550 , apto. 2603 - Bairro Tirol - CEP 59020160 - Natal (RN), Brasil - E-mail: patricia.foresmeyer@gmail.com
} 


\section{Introdução}

A origem do conceito de magnetismo é muito antiga, com mais de 3.500 anos, remontando ao meio da Idade do Ferro, no Egito antigo, China e Índia. Nos anos 131 a 201 a.C., o magnetismo foi utilizado para a cura de doenças inflamatórias e enfermidades dolorosas. Durante séculos, a magnetoterapia foi conhecida sob a forma de ímãs naturais, disponibilizando uma ampla variedade de propriedades, tais como prevenir ou retardar o processo de envelhecimento celular e manter a saúde. Estudos sobre as propriedades dos ímãs continuaram no século 16, com Aureolus Philippus Paracelso (1493-1541), que utilizou os ímãs para a cura de muitos processos inflamatórios. No fim do século 18, Faraday abriu caminho para uma nova concepção científica da associação da corrente elétrica e do magnetismo, estudando os efeitos dos campos eletromagnéticos ${ }^{1,2}$.

Os estudos relacionados aos campos eletromagnéticos aumentaram nos últimos 20 anos. Hoje, as nações industrializadas de todo o mundo, bem como as universidades e institutos científicos, empreendem a pesquisa no magnetismo e em sua exploração terapêutica. Entre os diversos tipos de tratamentos na área de saúde utilizando a magnetoterapia, existem os campos eletromagnéticos pulsados de baixa frequência (CEMP-BF), que são uma importante ferramenta da fisioterapia, tratando-se de um método não invasivo, seguro e de fácil manejo para o tratamento de diversas patologias caracterizadas, principalmente, por dor, inflamação e regeneração, causando efeitos biológicos em organismos, sistemas e órgãos ${ }^{3}$.

Existem muitas dúvidas em relação à melhor dosagem a ser utilizada com os campos eletromagnéticos pulsados (CEMP), sendo que os mecanismos de ação dos mesmos não estão totalmente esclarecidos ${ }^{3}$. Suas principais ações são o desvio de partículas com cargas elétricas em movimento, produção de correntes induzidas através do efeito piezoelétrico em ossos e tecido colágeno, e aumento da solubilidade de substâncias. Ao nível celular tem efeito normalizador do potencial de membrana e estimula o metabolismo celular; no tecido, é um potente estimulador metabólico de células, tecidos e órgãos ${ }^{4}$.

Portanto, os CEMP de baixa frequência são uma das terapias mais comumente utilizadas pelo fisioterapeuta para tratamento de pacientes com diferentes patologias e em diversos países, exceto no Brasil. Os CEMP - ou a magnetoterapia - não se tratam de uma técnica nova, mas são praticamente desconhecidos neste país. Devido ao longo tempo de utilização em todos os continentes e à quantidade de publicações existentes sobre o tema, realizou-se uma revisão de literatura a respeito para estimular a presença dessa técnica na rotina de tratamento das clínicas de fisioterapia brasileiras ${ }^{3,5}$. Sendo assim, este estudo objetivou investigar os efeitos e a aplicação clínica dos CEMP na rotina de tratamento fisioterapêutico.

\section{Métodos}

Iniciou-se esta pesquisa por meio de uma revisão sistemática na base de dados do Pubmed (http://www.ncbi.nlm.nih.gov/PubMed/) com as palavras-chave "PEMF", encontrando-se 274 resultados, e "Pulsed Electromagnetic Fields", encontrando-se 899 resultados. Considerou-se o período de 1968 a 2010 (42 anos). Após esse levantamento, foram selecionados 36 artigos do período de 1981 a 2008 hand made, além de consulta a 6 livros.

\section{Resultados e discussão}

Segundo o estudo epidemiológico de Desjobert et al..$^{5}$, a região com a maioria dos trabalhos sobre a magnetoterapia é a América do Norte, com quase $45 \%$ das investigações em relação ao total. Em seguida, encontra-se a Europa, com pouco mais de 41\%, e a Ásia e a Oceania, com pouco mais de $13 \%$. Segundo os mesmos autores, esses são os principais continentes que investigam a onda eletromagnética e a aplicação de campo magnético na Medicina e na Biotecnologia. O país mais envolvido na investigação da magnetoterapia são os Estados Unidos, com $53 \%$ das obras consultadas, seguido pela Alemanha, com 14\%, Japão, com 12\%, Reino Unido, com 11\%, e Canadá e Itália com 5\% do total.

Em relação à origem da magnetoterapia, seu uso terapêutico iniciou no final do século 19 com D’Ansorval, mas os estudos avançaram muito quando, em 1957, Fukuda e Yasuda descreveram o fenômeno piezoelétrico em tecido ósseo. A partir desses estudos, percebeu-se que os efeitos obtidos na regeneração óssea com CEMP não se devem apenas à energia mecânica aplicada aos íons de $\mathrm{Ca}^{++}$e que fazem vibrar esses íons em sua máxima frequência até chegar a ressonância (energia que será transformada em eletricidade pelo fenômeno piezelétrico), mas também às respostas dos osteócitos que liberam por via autócrina e parócrina os fatores moduladores da atividade celular ${ }^{6,7}$.

Martinez, Capellas e Tinoco ${ }^{8}$, estudando os efeitos da magnetoterapia em tecido ósseo, relatam que 28 sessões de magnetoterapia são necessárias para o tratamento de fratura de ossos longos. Nesse estudo, foi aplicada a magnetoterapia durante 30 minutos por dia a uma frequência de $50 \mathrm{~Hz}$ e intensidade de $8 \mathrm{mT}$. Seus resultados em relação à consolidação das fraturas foram confirmados radiologicamente.

O estudo clínico de McLeod et al. ${ }^{9}$ relata mais evidências a respeito, comparando os efeitos de 1 hora de CEMP de 15-75 e $150 \mathrm{~Hz}$ em um animal com osteopenia, e o resultado indicou que a frequência de $15 \mathrm{~Hz}$ foi a mais efetiva para aumentar a massa óssea.

Ainda discutindo a variabilidade de parâmetros na utilização dos CEMP em tecido ósseo, os CEMP de 4.444,44 Hz modulados a $15 \mathrm{~Hz}$, com uma intensidade de 1,6 mT, aumentaram a PGE, a TGF-bl (que diminui a reabsorção óssea dos osteoclastos e trata-se de um marcador para a diferenciação do processo de osteogêneses) e modularam o $\mathrm{NO}^{2-}$, diminuindo sua liberação na primeira fase do tratamento (quando se aumenta a concentração de $\mathrm{NO}^{2-}$, inibe-se a atividade de osteoblastos) e elevando a atividade da fosfatase alcalina ${ }^{10}$.

Outra pesquisa realizada por Chang et al. ${ }^{11}$ concluiu que 2 horas/ dia de exposição com 7,5 Hz e uma duração de pulso de $0,3 \mathrm{~ms}$, em diferentes intensidades, induzem a inibição ou ativação de osteoclastos. 
A análise da fratura de mandíbula em porcos, após tratamento utilizando magnetoterapia com cinco aplicações, comprovou uma osteogênese (crescimento lamelar na zona de fratura) mais rápida do que no grupo controle, o número de osteoblastos foi maior e a reação de macrófagos e neoangeogênese confirmou uma resposta de consolidação mais eficaz. Em cavalos, os CEMP na frequência de $75 \mathrm{~Hz}, 2,8$ $\mathrm{mT}$ (não foi descrita a forma de onda utilizada), obteve-se estímulo de reparação óssea e aumento da fase osteogênica. Um protocolo similar $(75 \mathrm{~Hz}, 1,6 \mathrm{mT})$ foi utilizado para acelerar a osteointegração da hidroxiapatita ${ }^{12-14}$.

Giordano et al. ${ }^{15}$ aplicaram durante 1 hora (3 vezes por semana durante 3 meses) CEMP de $100 \mathrm{~Hz}$ com componentes estáticos e sinusoidais em mulheres em pós-menopausa com osteoporose, observando um aumento significativo da osteocalcina sérica do propéptido sérico pró-colágeno tipo I C-terminal, indicadores do aumento da atividade dos osteoblastos e estimulação osteogênica.

Os CEMP também trabalham a estimulação celular e a regeneração nervosa. Sisken et al. ${ }^{16}$ estudaram os efeitos dos CEMP na lesão nervosa do nervo ciático de ratos expostos à frequência de $2 \mathrm{~Hz}, 0,3 \mathrm{mT}$ durante 3-6 dias, por 4, 1 e 10 horas/dia. Os resultados encontrados demonstraram o aumento da regeneração nervosa em $22 \%$ dos casos, independentemente do tempo de exposição. Esses efeitos podem ser explicados por meio da ação dos CEMP no nível dos fatores de crescimento, promovendo regeneração nervosa pós-lesão e incrementando a queda precoce da atividade do $\mathrm{NGF}^{17}$.

Os CEMP podem auxiliar no tratamento da osteoartrite, desencadeando a diminuição da dor com aplicações de 0,1-64 Hz, $30 \mathrm{~min} /$ dia durante 3 semanas, preservando a morfologia da cartilagem e retardando o desenvolvimento de lesões osteoartríticas por meio da supressão de enzimas de degradação da matriz colágena ${ }^{18-20}$.

Alguns artigos relatam o incremento e o crescimento diferenciado dos condrócitos, provavelmente devido ao aumento do Transforming Growth Factors (TGFa) e ao Insulin Grow Factor (IGF), que são ativadores essenciais desse processo. $\mathrm{O}$ incremento do fluxo sanguíneo e a angiogênese, estimulados pelos CEMP, também auxiliam na nutrição da cartilagem, sendo que esses efeitos ocorreram nos parâmetros de $75 \mathrm{~Hz}$, com intensidades desde 1,5 até $2,3 \mathrm{mT}$ e foram realizados in vitro ${ }^{1,21-27}$.

$\mathrm{O}$ efeito anti-inflamatório é fundamental para diversas patologias, como as inflamações crônicas, osteoartrite, artrite reumatoide e psoríases, tendinites, mas os CEMP não apresentam bom resultado em inflamações causadas por bactérias. Em $50 \mathrm{~Hz}$, os CEMP produzem apoptoses dos linfócitos $\mathrm{T}^{27,28}$.

Em pacientes com epicondilite, após 15 sessões de 30 minutos de exposição a $6 \mathrm{mT}, 25-46 \mathrm{~Hz}$, ocorreu redução do quadro de dor comparado ao grupo placebo. Em uma tendinite de Aquiles induzida em ratos utilizando-se a frequência de $50 \mathrm{~Hz}$ e uma forma de onda retificada, obteve-se diminuição significativa do edema e produção de fibroblastos em maior quantidade, além do alinhamento do tecido cicatricial detectado ao se utilizar a frequência de $17 \mathrm{~Hz}^{22,30}$.
Em todas as patologias em que a aceleração do processo de recuperação celular é necessária, existe a aplicabilidade da magnetoterapia, graças à rápida cinética dos processos induzidos por esse agente físico ${ }^{30}$. Encontram-se também resultados comprovados nas úlceras, por estimulação da produção de fibroblastos e controle de infecções ${ }^{31-33}$. Em um estudo, foram utilizados $40 \mathrm{~Hz}$ e $10 \mathrm{mT}$ durante 10 dias de tratamento, e a avaliação foi realizada pela medição da quantidade de hidroxiprolina na ferida e microscopia eletrônica, demonstrando superioridade de resultados e incremento da matriz colágena no grupo tratado comparado ao controle $\mathrm{e}^{34,35}$.

Avaliando-se seus efeitos biológicos, foi estudada a sua ação no sangue em relação à extensão do tempo de atuação da protrombina, diminuição da atividade do fator Xa e decréscimo do nível de plaquetas, que foram observados em experimento com animais após a exposição aos CEMP de baixa frequência, concluindo que essa fonte causa mudanças na coagulação sanguínea ${ }^{36}$.

Os CEMP são capazes de influenciar positivamente inúmeros processos bioquímicos: orientação molecular, atividade enzimática, interação oxigênio-substrato, transporte de membrana, síntese de colágeno, síntese do ácido desoxirribonucleico, produção de endorfinas, inibição de radicais livres e aumento no número de mitoses de células de cultivo ${ }^{37}$.

Esses efeitos dos CEMP são corroborados na pratica diária, mas as variáveis de melhor dosificação não foram totalmente esclarecidas. Novos estudos têm surgido a cada dia, abordando tempos de exposição bastante extensos e baixas intensidades de fluxo magnético; porém, na prática clínica, percebe-se que usualmente se aplica entre 30-45 min/ paciente com uma intensidade elevada. Alguns autores sugerem que se devem evitar exposições prolongadas devido à produção de radiciais livres. Foram analisados os efeitos dos CEMP por 8 horas, $2 \mathrm{mT}$ e $50 \mathrm{~Hz}$ em ratos, detectando-se, com essas doses, danos oxidativos. Deve-se considerar o peso do animal ao ser comparado ao adulto humano; portanto, os achados deste estudo podem ser questionados ${ }^{38}$. Portanto, a literatura, de maneira geral, apresenta informações incompletas sobre os parâmetros de utilização da magnetoterapia (Quadro 1).

O tratamento com a magnetoterapia está contraindicado em casos de suspeita ou confirmação de processos neoplásicos, hiperfuncionalidade das glândulas tireoide e suprarrenais, miastenia grave, hemorragias e em casos de infecções fúngicas e virais, visto que o tratamento pode acelerar o processo de proliferação celular, aumentando o quadro infeccioso ${ }^{39}$.

Segundo Quintero ${ }^{40}$, deve-se tomar algumas precauções especiais na utilização dos CEMP como, por exemplo, o uso de marcapasso pelos pacientes - visto que o campo magnético poderia alterar o ajuste do ritmo elétrico desse aparelho quando submetido a proximidades de regiões cardíacas. Os CEMP também podem interferir no período gestacional, além de provocar alguma lesão de pele, como feridas infeccionadas, sangramentos ou hemorragias. Devem-se evitar tratamentos associados a enfermidades neurológicas com ataques epiléticos, 
Quadro 1 - Protocolos de magnetoterapia

\begin{tabular}{|lllc|}
\hline Frequência $(\mathrm{Hz})$ & Tempo & Efeito & Referência \\
\hline 0,1 a 64 & 30 minutos/dia & Analgesia em osteoartrite & $18-20$ \\
7,5 & 2 horas/dia & Osteoregeneração & 11 \\
15 & 1 hora & Osteoregeneração & 9 \\
16 & 1 hora ou mais/dia & Osteoregeneração & 6,7 \\
50 & 30 min/3 vezes por semana & Osteoregeneração & 8 \\
16 & 30 minutos/dia & Alinhamento de colágeno & 30 \\
2 & 1 hora/dia & Incremento da regeneração nervosa & 16 \\
$25-46$ & 30 minutos/dia & Analgesia em epicondilite & 29 \\
50 & 4 horas/dia & Incremento da regeneração nervosa & 16 \\
75 & 6 horas/dia & Proteção e regeneração de condrócitos & 21,22 \\
75 & 24 horas & Angiogênese & 24 \\
50 & 48 Horas/dia ${ }^{27}$ & Anti-inflamatório & 27,28 \\
100 & Acima de 30 minutos/dia ${ }^{28}$ & & 15 \\
\hline
\end{tabular}

arteriosclerose grave, ciclo menstrual, hipotensão arterial, pacientes anticoagulados, hipotônicos e psicopatas.

\section{Considerações finais}

Após o levantamento dos efeitos da magnetoterapia, foram observados resultados positivos no tecido ósseo em relação à consolidação de fraturas e à osteoporose, devido à sua ação piezoelétrica. Foram observados também nos resultados efeitos positivos em relação à cicatrização, regeneração nervosa e proteção e regeneração da cartilagem articular, que poderiam ser inlcusos nas considerações finais.
O conhecimento desse recurso no Brasil deve ser despertado em todos os profissionais de saúde que compõem as equipes multidisciplinares de assistência, para que passe a fazer parte da rotina de trabalho dos fisioterapeutas, beneficiando os pacientes portadores de diferentes problemas.

Sugere-se um incentivo à produção nacional desse tipo de agente físico, a fim de que o custo e o acesso a esse tratamento sejam facilitados e para que possa estar presente em serviços públicos e privados. Diante de tantos efeitos descritos, as empresas poderão produzir para atender a mercados diferenciados, dentro das especialidades da saúde, auxiliando o profissional e o paciente.

\section{Referências}

1. Díaz DS, Mulens IF, Mahojo LAF, Aguilera SG, Rodríguez ZP. Magnetoterapia en el tratamiento de la neuropatía óptica epidémica. Rev Cubana Oftalmol. 1995;8:(1)13-7.

2. Guillot JDZ. La magnetoterapia y su aplicación em la medicina. Rev. Cubana Med Gen Integr. 2002;18(1):60-72.

3. Meyer PF. Investigação sobre possíveis efeitos biológicos in vitro de agentes físicos utilizados em fisioterapia. Tese (Doutorado) - Universidade Federal do Rio Grande do Norte, Natal: 2008; p.1-52.

4. Zaragoza J. Física e instrumentación médicas. $2^{\mathrm{a}}$ ed. Barcelona: Ediciones Científicas y Técnicas; 1992.

5. Desjobert $\mathrm{H}$, Hillion J, Adolphe $\mathrm{M}$, Averlant G, Nefziger J. Effects of $50 \mathrm{~Hz}$ magnetic fields on C-MYC transcript levels in nonsynchronized human cells. Bioelectromagnetics. 1995; 16:277-83.

6. Ronzio OA Capponi RID. Magnetoterapia: un acercamiento a sus efectos y dosificación. Rev Cient Col Kinesiólogos Prov Buenos Aires. 2008; 7(27): 19-25.

7. Fukada E, Yasuda I. On the piezoelectric effect of bone. J Physiol Soc Japan. 1957; 12:1158-62
8. Martínez Escudero C, Capellas Sans L, Tinoco Gonzáles J. Magnetoterapia en retardos de consolidación. Rehabilitación. 2001;35(5):312-4.

9. McLeod KJ, Rubin CT. The effect of low-frequency electrical fields on osteogenesis. J Bone Joint Surg Am. 1992;74A:920-9.

10. Bodamyali T, Bhatt B, Hughes FJ, Winrow VR, Kanczler JM, Simon B et al. Pulsed electromagnetic fields simultaneously induce osteogenesis and upregulate transcription of bone morphogenetic proteins 2 and 4 in rat osteoblasts in vitro. Biochem Biophys Res Common. 1998;250(2):458-61.

11. Chang K, Hong-Shong Chang W, Huang S, Huang S, Shih C. Pulsed electromagnetic fields stimulation affects osteoclast formation by modulation of osteoprotegerin, RANK ligand and macrophage colonystimulating facto. J Orthop Res. 2005;23(6)130814.

12. Faustov LA, Nedel'ko NA, Morozova MV. Pathomorphology of regenerative processes in mandibular fracture after sodium succinate treatment and laser magnetotherapy in an experimental setting. Stomatologiia. 2001;80(6): 8-11.

13. Bromiley MW. Equine injury: therapy and rehabilitation. Oxford: Blackwell Scientific; 1993 
14. Fini M, Cadossi R, Canè V, Cavani F, Giavaresi G, Krajewski A et al. The effect of pulsed electromagnetic fields on the osteointegration of hydroxyapatite implants in cancellous bone: a morphologic and microstructural in vivo study. J Orthop Res. 2002;20(4):756-63.

15. Giordano N, Battisti E, Geraci S, Fortunato M, Santacroce C, Rigato M et al. Effect of electromagnetic fields on bone mineral density and biochemical markers of bone turnover in osteoporosis: a single-blind, randomized pilot study. Current Therapeutic Research. 2001;62(3):187-93.

16. Sisken BF, Jacob JM, Walker JL. Acute treatment with pulsed electromagnetic fields and its effect on fast axonal transport in normal and regenerating nerve. J Neurosci Res. 1995;42(5):692-9.

17. Longo FM, Yang $T$, Hamilton $S$, Hyde JF, Walker J, Jennes $L$ et al. Electromagnetic fields influence NGF activity and levels following sciatic nerve transection. J Neurosci Res. 1999;55(2):230-7.

18. Sutbeyaz ST, Sezer N, Koseoglu BF. The effect of pulsed electromagnetic fields in the treatment of cervical osteoarthritis: a randomized, double-blind, sham-controlled trial. Rheumatol Int. 2006;26(4):320-4.

19. McCarthy CJ, Callaghan MJ, Oldham JA. Pulsed electromagnetic energy treatment offers no clinical benefit in reducing the pain of knee osteoarthritis: a systematic review. BMC Musculoskeletal Disorders. 2006;7:51.

20. Thamsborg G, Florescu A, Oturai P, Fallentin E, Tritsaris K, Dissing S. Treatment of knee osteoarthritis with pulsed electromagnetic fields: a randomized, double-blind, placebo-controlled study. Osteoarthritis Cartilage. 2005;13(7):575-81.

21. Pezzetti F, De Mattei M, Caruso A, Cadossi R, Zucchini C, Carinci F et al. Effects of pulsed electromagnetic fields on human chondrocytes: an in vitro study. Calcif Tissue Int. 1999; 65(5):396-401.

22. De Mattei M, Caruso A, Pezzetti F, Pellati A, Stabellini G, Sollazzo V et al. Effects of pulsed electromagnetic fields on human articular chondrocyte proliferation. Connect Tissue Res. 2001;42(4):269-79.

23. Smith TL, Wong-Gibbons D, Maultsby J. Microcirculatory effects of pulsed electromagnetic fields. J Orthop Res. 2004;22(1):80-4.

24. Tepper OM, Callaghan MJ, Chang El, Galiano RD, Bhatt KA, Baharestani S et al. Electromagnetic fields increase in vitro and in vivo angiogenesis through endothelial release of FGF-2. FASEB J. 2004;18(11):1231-3.

25. Fini M, Giavaresi G, Carpi A, Nicolini A, Setti S, Giardino R. Effects of pulsed electromagnetic fields on articular hyaline cartilage: review of experimental and clinical studies. Biomed Pharmacother. 2005;59(7):388-94.

26. De Mattei M, Pasello M, Pellati A, Stabellini G, Massari L, Gemmati D et al. Effects of electromagnetic fields on proteoglycan metabolism of bovine articular cartilage explants. Connect Tissue Res. 2003;44(3-4):154-9.
27. Nindl G, Johnson MT, Balcavage WX. Low frequency electromagnetic field effects on lymphocytes: potential, for treatment of inflammatory diseases. Disponível em: http://www.immunent.com/forum/showthread.php?t=225. Acesso em 17 mar. 2011.

28. Varani K, Gessi S, Merighi S, lannotta V, Cattabriga E, Pancaldi $C$ et al. Alteration of $A(3)$ adenosine receptors in human neutrophils and low frequency electromagnetic fields. Biochem Parmacol. 2003;66(10):1897-906.

29. Uzunca K, Birtane M, Taştekin N. Effectiveness of pulsed electromagnetic field therapy in lateral epicondylitis. Clin Rheumatol. 2007;26(1):69-47.

30. Lee EW, Maffulli N, Li CK, Chan KM. Pulsed magnetic and electromagnetic fields in experimental achilles tendonitis in the rat: a prospective randomized study. Arch Phys Med Rehabil. 1997;78(4):399-404.

31. Espinosa Álvarez RF, Montero García, JL, Novoa Blanco, JF. Tratamiento magnético de los traumatismos no complicados. Reportes preliminares. Rev Cubana Med Gen Integr. 2007;23(4):1-6.

32. Kul'chitskaia DB, Orekhova EM, Vasil'eva ES. Using combined magnetotherapy in patients with acne. Vopr Kurortol Fizioter Lech Fiz Kult. 2004;(4):28-30.

33. Nötzli HP, Swiontkowski MF, Thaxter ST, Carpenter GK, Wyatt R. Laser doppler flowmetry for bone blood flow measurements: Helium-neon laser light attenuation and depth of perfusion assessment. J Orthop Res. 1989;7(3):413-24.

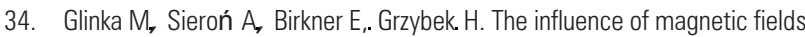
on the primary healing of incisional wounds in rats. Electromagnetic Biology and Medicine. 2002;21(2):169-84.

35. Leaper DJ, Foster ME, Brennan SS; Davies PW, Do magnetic fields influence soft tissue wound healing? A preliminary communication. Equine Vet J. 1985;17(3):178-80.

36. Bromiley MW. Equine injury, therapy and rehabilitation. Oxford: Blackwell Scientific; 1993.

37. Sodi-Pallares D. Magnetoterapia y tratamiento metabólico. Mexico: c1994.

38. Eraslan G, Bilgili A, Akdogan M, Yarsan E, Essiz D, Altintas L. Studies on antioxidant enzymes in mice exposed to pulsed electromagnetic fields. Ecotoxicol Environ Saf. 2007;66(2):287-9.

39. Silva RMV, Costa LS, Meyer PF. Efeitos da magnetoterapia na cicatrização do tecido muscular (Trabalho de Conclusão de Curso) - Universidade Potiguar de Natal, Natal, 2010.

40. Quintero HC, Hernández DR, Madan GH, Lio LN, Ramírez MD. Agentes físicos en el tratamiento de la enfermedad pulmonar obstructiva crónica. Rev Cubana Med Milit. 2002;31(2):119-25. 